\title{
CDISC SDTM Pharmacogenomics Biomarker Medical Statement Terminology
}

National Cancer Institute

\section{Source}

National Cancer Institute. CDISC SDTM Pharmacogenomics Biomarker Medical

Statement Terminology. NCI Thesaurus. Code C114119.

Terminology associated with the pharmacogenomics biomarker medical statement codelist of the Clinical Data Interchange Standards Consortium (CDISC) Study Data Tabulation Model (SDT M). 